\title{
Brief Papers
}

\section{Robust Tuning of Power System Stabilizers Using QFT}

\author{
P. Shrikant Rao and Indraneel Sen
}

\begin{abstract}
This paper presents a new method for tuning the parameters of a conventional power system stabilizer. The region of acceptable performance for the stabilizer has been extended and covers a wider range of operating and system conditions. The parametric uncertainty in power systems has been handled using quantitative feedback theory (QFT). The required controller parameters are arrived at by solving an optimization problem that incorporates the control specifications. The robustness of the feedback controller has been investigated on a single machine infinite bus model and the results are shown to be consistent with the expected performance of the stabilizer.
\end{abstract}

Index Terms-Parameter optimization, power system dynamic stability, power system stabilizers, quantitative feedback theory, robust controllers.

\section{INTRODUCTION}

$\mathbf{T}$ HE PROBLEM of low-frequency oscillatory instability of power systems has been the topic of much research in the last few decades [10]. One of the cost effective solutions to the problem is fitting the generators with a feedback controller to inject a supplementary signal at the voltage reference input of the automatic voltage regulator to damp the oscillations. This device, known as a power system stabilizer (PSS), is now widely used in the electric power industry. Over the years, several innovative methods have evolved for designing these stabilizers. Amongst these, the conventional lead compensation type of PSS [7] has been the most popular with power utilities because of its fixed gains and operational simplicity. Properly tuned, these PSS's can considerably enhance the dynamic performance of a system. Tuning these stabilizers is not easy however due to the constantly changing nature of power systems. There has been some effort in designing self-tuning and adaptive power system stabilizers [3], [13] to account for these variations. However, the complexity and real-time computational requirements preclude their usage in actual power plants. Recent progress in the field of robust control has given a new impetus for designing fixed gain controllers that in theory perform well in spite of parametric variations in the system. The design method proposed in this paper follows a similar approach.

Power systems constantly experience changes in the operating condition due to variations in generation and load patterns, as well as changes in the transmission network. As a result,

Manuscript received January 24, 1997; revised January 28, 1998. Recommended by Associate Editor, J. Hung.

The authors are with the Department of Electrical Engineering, Indian Institute of Science, Bangalore 560 012, India.

Publisher Item Identifier S 1063-6536(99)05250-1. there is a corresponding large variation in the small signal dynamic behavior of the system. This can be expressed as a parametric uncertainty in the small signal linearized model of the plant. The tuning problem, therefore, reduces to choosing a set of controller parameters such that the system is well damped in spite of changes in the operating condition, i.e., changes in some parameters of the linearized model of the plant to be controlled.

The conventional PSS design deals with a single, properly chosen, nominal operating condition and attempts to optimize the controller performance for the small signal linearized model of the plant about this operating point [6], [7]. Optimization in these cases generally implies the improvement of the system damping which is captured in an aptly defined performance index. This approach implicitly assumes that maximizing the damping at the nominal operating point is likely to give a large region around the nominal point, in which, at least, an acceptable performance in terms of damping is achieved. Whether this is actually so depends upon the way in which the plant parameters change with operating condition. An alternative and more logical approach would be to first specify the acceptable range of performance and then attempt to obtain a controller which achieves this specification over the required range of operating conditions.

\section{Performance ReQuirements OF THE POWER SySTEM STABILIZER}

In power systems, a damping factor $\zeta$ of around 10 to $20 \%$ for the low-frequency electromechanical mode is adequate. (For a second order system, $\zeta=10 \%$ implies system oscillations decaying to within $15 \%$ of the initial amplitude in three cycles.) In addition, if the real parts of the dominant poles are restricted to be less than a specified value, say $\alpha$, it would guarantee a minimum rate of decay. The closed-loop rotor modes must satisfy these two requirements simultaneously for an acceptable performance. The frequency of oscillations is related to the synchronizing torque of the generators and hence care should be taken to ensure that the imaginary part of the rotor mode eigenvalue does not fall appreciably due to the feedback. Any controller which satisfies these conditions on the closed-loop rotor mode eigenvalue is acceptable. Any new modes arising as a result of closing the controller loop (e.g., exciter mode) should also be well-damped. Presence of real poles close to the origin results in a sluggish response and persistent deviation of the system variables from their steady-state values and 


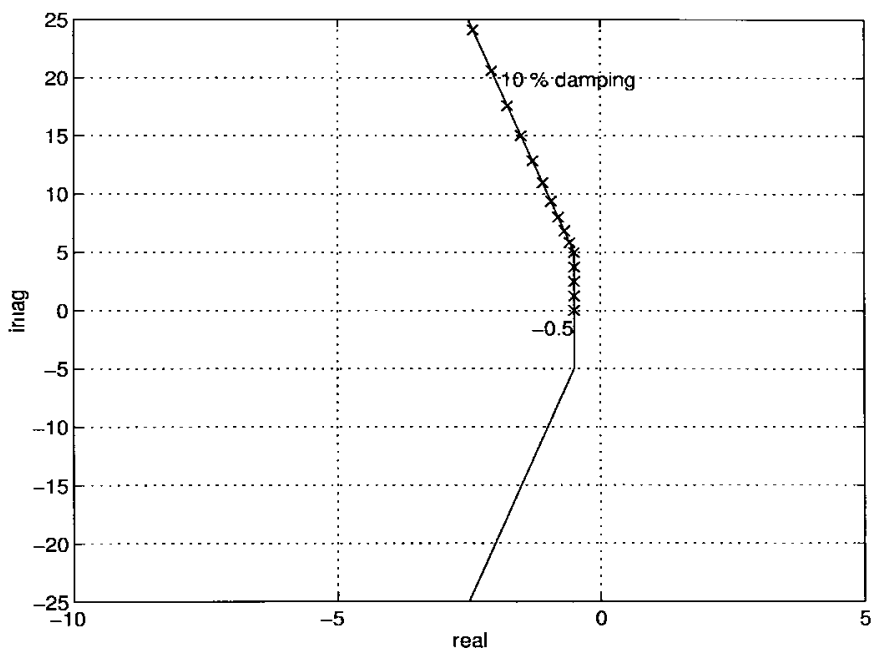

Fig. 1. The $D$ contour.

should be avoided. Finally, a small-loop gain is desirable to avoid possible controller saturation and poor large disturbance response.

The above discussion suggests rotor mode or dominant pole region location as an effective performance criterion in PSS design. If all the closed-loop poles can be placed to the left of the " $D$ " contour shown in Fig. 1, then the specified damping requirements are satisfied. This property will be henceforth referred to as " $D$-stability." Any PSS that achieves $D$-stability for a given range of operating conditions is said to be robust, i.e., it guarantees an acceptable performance over that range of operating conditions.

The main difficulty in designing such controllers lies with the magnitude of uncertainty. In power systems the model uncertainties are typically large, and most robust controller design methods that incorporate a conservative description of the uncertainty often fail to provide a solution even when one exists. Many of the recently developed robust control theories also suffer from this drawback. Frequency domain techniques, such as, $H_{\infty}$-optimization and $\mu$-synthesis do not provide much control over the closed-loop pole location and hence the transient response of the system, the main objective of the power system stabilizer design. Quantitative feedback theory (QFT), on the other hand, does not introduce any conservativeness in the uncertainty description and is therefore more likely to provide a solution with acceptable system performance [4], [5].

The following section discusses the QFT approach of designing a stabilizer to achieve robust $D$-stability, that is, $D$-stability for a set of plants or over a range of operating conditions.

\section{PSS DESIGN BASED ON QFT}

The discussion here covers only those aspects of QFT that are relevant to the proposed PSS design method, which is a slight deviation from the methodology originally suggested by Horowitz and Sidi [5]. In QFT, the closed-loop transfer function needs to satisfy certain performance requirements for a set of discrete frequencies. These requirements are specified

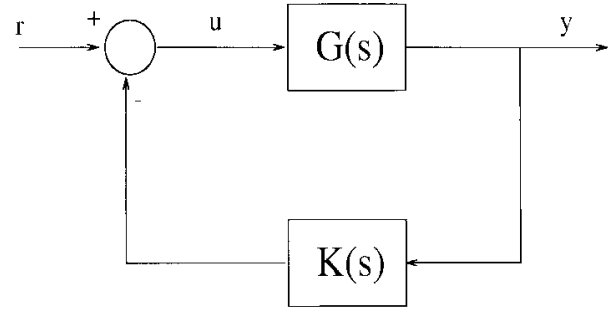

Fig. 2. One degree of freedom controller scheme.

in terms of tolerance bands within which the magnitude response of the closed-loop transfer function should lie. The uncertainties in the plant are transformed onto the Nichols chart resulting in bounds on the loop transmission function of an arbitrarily chosen, nominal plant. A compensator is chosen by manually shaping the loop transmission such that it satisfies the bounds at each of the frequency points. A prefilter is then used to ensure that the closed-loop transfer function lies within the specified bands.

In this paper the focus is only on achieving robust $D$ stability and not on shaping system transfer functions. The conventional QFT procedure can be used to design a controller for a system subject to parametric uncertainty, which achieves robust stability of the closed-loop. Here, the same basic principle is used to achieve robust $D$-stability by working with a set of discrete complex frequency points chosen on the $D$ contour instead of the imaginary axis. A controller transfer function satisfying the resulting constraints at the chosen, complex frequency points is then synthesized using parameter optimization.

Consider the configuration shown in Fig. 2. $G(s)$ is the plant, which, due to the uncertainty in the plant parameters, is only known to belong to a set, $\mathcal{G}$, of plants. The value set $G\left(j \omega_{i}\right), G \in \mathcal{G}, \omega_{i} \in \mathcal{R}$, is called the "plant template" at frequency $\omega_{i}$. A template thus represents the range of variations in the plant response at a particular frequency $\omega_{i}$.

Plant templates can be plotted on the Nichols chart at any desired frequency by computing $G(j \omega)$ as $G$ varies over the set $\mathcal{G}$ and then manually constructing a boundary around the set of points thus obtained. The introduction of the controller $K(s)$, shifts the template at each frequency to a new location on the Nichols chart without altering its shape. This shift depends upon the value of $K(s)$ at that frequency. Fig. 3 shows a typical plant template at its original location as also in the shifted location after the introduction of the controller. Each point on this plot represents a computed value of $G(s)$ as $G$ is varied over the set $\mathcal{G}$. The boundary of the template is approximated by straight line segments and is drawn manually by inspection. One plant in $\mathcal{G}$, designated $G_{o}$, is arbitrarily chosen as the nominal plant.

The closed loop will be robustly stable (i.e., stable $\forall G \in \mathcal{G}$ ) if the following three conditions are satisfied.

1) The templates of the compensated plant $(K(s) G(s))$ do not contain the point $\left(-180^{\circ}, 0 \mathrm{~dB}\right)$ on the Nichols chart $\forall \omega \in \mathcal{R}$.

2) The set $\mathcal{G}$ is connected.

3) The nominal closed loop is stable. 


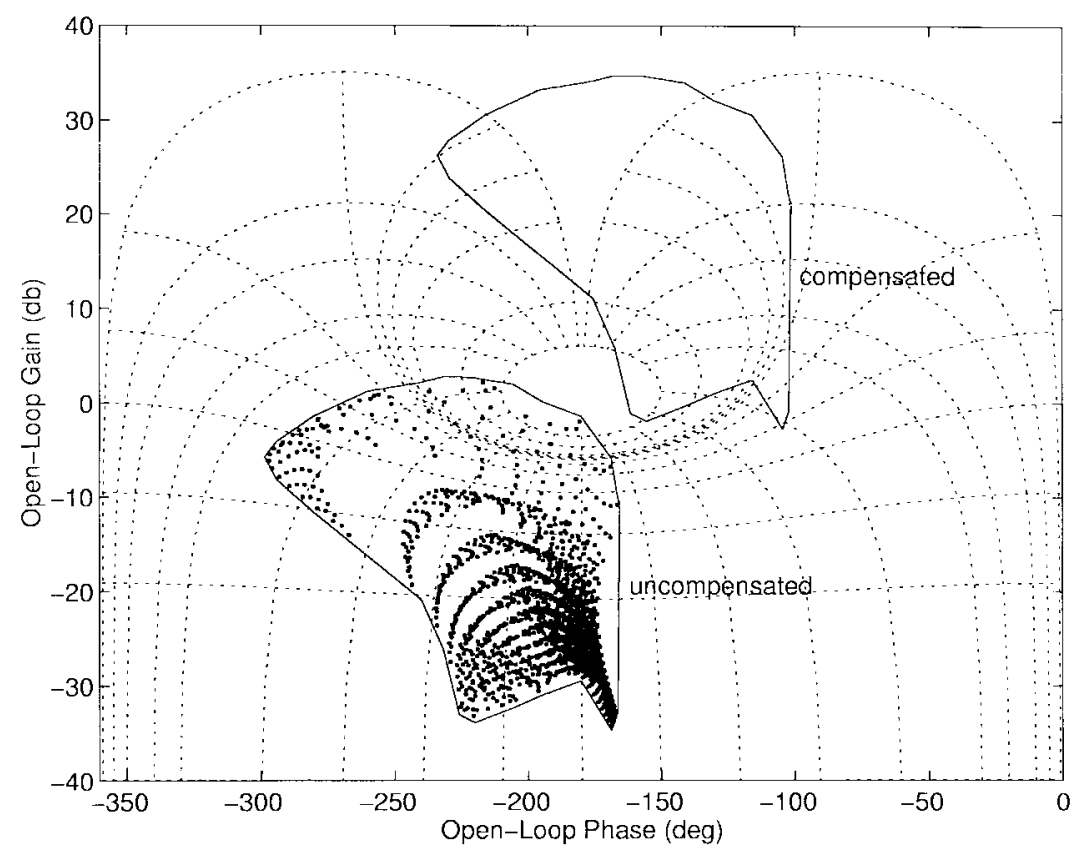

Fig. 3. A plant template at frequency $\omega_{i}$ and its shift due to compensation.

Any controller that satisfies the above three conditions robustly stabilizes the given connected set of plants. These conditions can be extended to the case of $D$-stability with the only modification being that (1) should be satisfied for all points on the $D$-contour instead of points on the imaginary axis. The plant templates are, therefore, drawn for the points on the $D$-contour. The templates are then shifted by choosing $K(s)$ such that none of the templates contain the point $\left(-180^{\circ}\right.$, $0 \mathrm{~dB})$. In addition, if the nominal closed loop is $D$-stable, then robust $D$-stability for the set of plants is achieved. The procedure for designing a robustly $D$-stabilizing controller for the given set of plants can be summarized as follows.

1) Choose a set of points on the $D$-contour, for which the robust stability is desired. These points should be closely spaced in the frequency range of interest and in regions where there is a rapid change in the frequency response of the plant.

2) Plot the plant templates for each of the chosen set of points. For this, $G(s)$ is computed as $G$ is varied over $\mathcal{G}$, in steps small enough to give clearly defined template boundaries. The template boundaries are then drawn manually.

3) Choose a controller for which the nominal closed loop is $D$-stable and all the compensated templates avoid the point $\left(-180^{\circ}, 0 \mathrm{~dB}\right)$ on the Nichols chart.

Interactive shaping of the frequency response of the controller, as originally suggested by Horowitz [4], [5], would be impracticable in the present case. An alternative method could be based on parameter optimization. The requirements stated above, can be formulated as constraints. One can then solve a goal attainment or optimization problem in the controller parameters such that the controller satisfies the specified constraints.
In QFT, the satisfaction of the critical point exclusion constraint is considered only at a discrete set of frequencies. In some cases, it is therefore possible that robust $D$-stability of the closed loop is not achieved in spite of all the templates avoiding the critical point. In such cases, the number of points on the $D$-contour chosen for the design can be increased by considering additional points that are close to the frequencies of the unstable eigenvalues.

\section{The Power System Model}

A single line representation of a single machine infinite bus (SMIB) power system is shown in Fig. 4(a). The generator is fitted with an automatic voltage regulator (AVR) and a static excitation system. For small oscillation stability analysis, the fast stator transients can be neglected. The $d$ and $q$ axis damper windings always produce positive damping and therefore are often not included for performance analysis of power system stabilizers. With these assumptions, the generator and excitation system can be modeled as a fourth-order system with changes in the load angle $-\Delta \delta$, the rotor speed $-\Delta \omega$, the internal voltage of the generator $-\Delta E_{q}^{\prime}$, and the field voltage $-\Delta E_{F D}$, as the state variables. The set of equations governing the behavior of this system is given in Appendix I-A. The system data for the chosen example is given in Appendix I-B. The details of the modeling procedure and the implications of the simplifying assumptions are discussed more completely in [10]. The linear time invariant model for this system is constructed by linearizing the system equations about any given steady-state operating condition. The input to this system is the AVR voltage reference and its output is the rotor slip - the signal to be fed back by the controller. The block diagram of the linearized system with the feedback loop is shown in Fig. 4(b) [10]. 


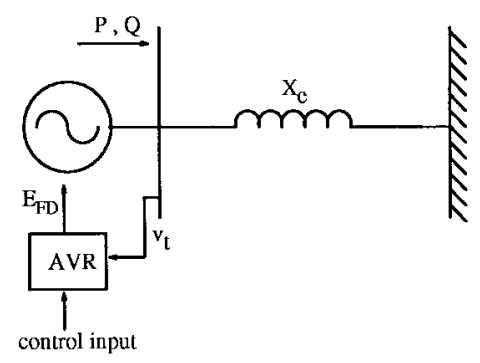

(a)

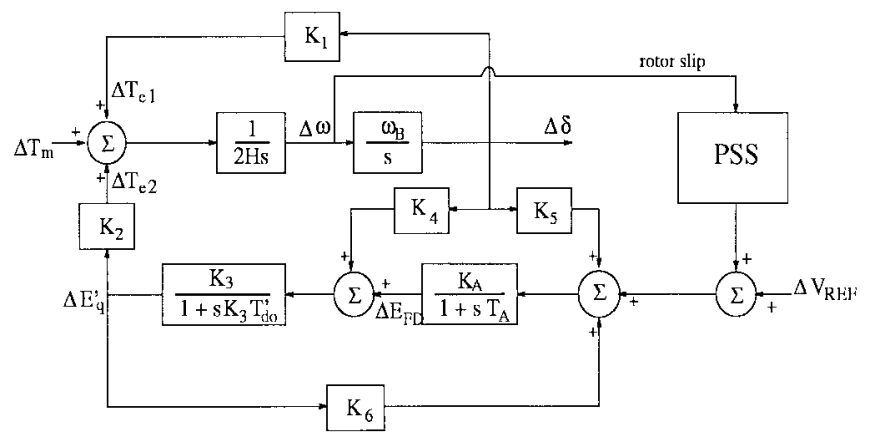

(b)

Fig. 4. Single machine infinite bus power system model. (a) Single line representation. (b) Block diagram of the linearized system and controller (PSS).

The operating condition for the above system is completely defined by the values of the real power $P$ and the reactive power $Q$ at the generator terminals and the impedance of the transmission line $X_{e} . P, Q$, and $X_{e}$ are assumed to vary independently over the range $P: 0.4$ to $1.0, Q:-0.2$ to 0.5 , and $X_{e}: 0.2$ to 0.7 . This encompasses almost all practical operating conditions for the generator and very weak to very strong transmission systems as represented by the varying lengths of the transmission lines. Since this set in the $\left(P, Q, X_{e}\right)$ space is connected and the elements of the $A$ matrix of the linearized system are continuous functions of $P, Q$, and $X_{e}$, the set of plants represented by this set is connected.

For designing the stabilizer, the $D$-contour is constructed with $\zeta=10 \%$ and $\alpha=-0.5$ as shown in Fig. 1. This represents the minimum acceptable damping requirements for the rotor mode. The points marked with crosses are chosen for the QFT based design. The plant corresponding to $P=0.5$, $Q=0.0$, and $X_{e}=0.2$ is arbitrarily chosen as the nominal plant. In many design procedures the choice of the nominal plant can be crucial, needing an "engineering judgement." This choice is less critical in the proposed method as the solution is not based on the attainment of the global minima. Any solution that places the closed-loop plant poles to the left of the $D$ contour is acceptable. To determine the system performance without any controller, $P, Q$, and $X_{e}$ are varied independently over the specified ranges with a step size of 0.05 . The choice of step size is arbitrary and is chosen to produce clearly defined templates needed for the design of the controller. Fig. 5 is the plot of plant poles, as the operating condition varies over the chosen set. It can be seen, that without a controller, the system is not $D$-stable for all operating conditions and is also

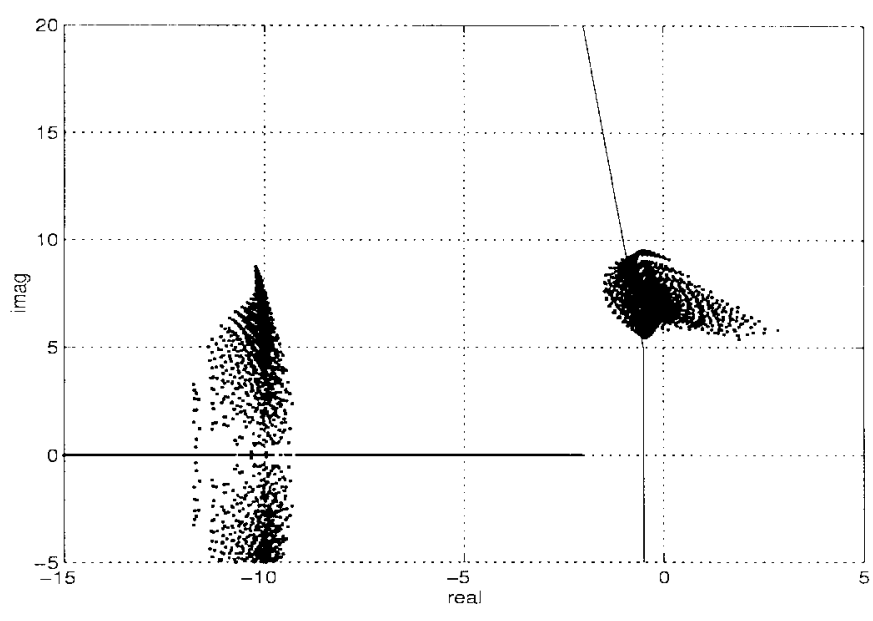

Fig. 5. Plant poles for the set considered.

unstable at some of the operating points. It is required to design a controller for this system which robustly places the closed loop poles to the left of the $D$-contour.

\section{CONTROLler Synthesis}

A controller satisfying requirements (1) and (3) is obtained by first choosing a particular structure for the controller and then solving an optimization problem to compute the controller parameters.

The problem is formulated by defining a set of constraint functions $g_{i}$, such that $g_{i}>0$ if the $i$ th compensated template includes the point $\left(-180^{\circ}, 0 \mathrm{~dB}\right)$ and $g_{i}<0$ otherwise. Hence, $g_{i}<0$ implies that there are no closed-loop poles at the $i$ th frequency point for the entire set of plants.

Let $d_{i}$ be the minimum distance of the point $\left(-180^{\circ}, 0\right.$ $\mathrm{dB})$ from the boundary of the $i$ th compensated template. The value of $d_{i}$ can be easily computed given any instance of the controller transfer function. Then $g_{i}$ can be defined as

$$
\begin{aligned}
g_{i}=+d_{i} ; & \text { if the point }\left(-180^{\circ}, 0 \mathrm{~dB}\right) \text { lies outside the } \\
& i \text { th compensated template. } \\
=-d_{i} ; & \text { if the point }\left(-180^{\circ}, 0 \mathrm{~dB}\right) \text { lies inside the } \\
& i \text { th compensated template. }
\end{aligned}
$$

To enforce $D$-stability of the nominal closed loop, two additional constraints are required

$$
\begin{aligned}
& g_{n+1}=\max \operatorname{Real}\left(\lambda_{i}\right)-\alpha \\
& g_{n+2}=\zeta-\max \left(\operatorname{Damp}\left(\lambda_{i}\right)\right)
\end{aligned}
$$

where $\lambda_{i}$ are the poles of the nominal plant with controller. Real( ) represents the real part and Damp( ) the damping factor of the argument. Constraining $g_{n+1}, g_{n+2}<0$ ensures that the nominal closed loop is $D$-stable. The problem now reduces to that of finding a controller such that $g_{i}<0 \forall i$, i.e.,

$$
\text { Find } x \text { s.t. } g_{i}(x)<0, \quad i=1,2, \cdots, n+2
$$

where $x$ is a vector of controller parameters. 


\section{IMPLEMENTATION AND RESULTS}

A PSS provides additional damping by producing a component of electrical torque on the rotor shaft which is in phase with the rotor oscillation. To achieve this, the stabilizing signals and the transfer function of the stabilizer have to be properly selected in order that both the gain and the phase characteristics of the excitation system, the synchronous generator, and the power system can be effectively compensated by the damping controller [12].

One of the most commonly used stabilizing signals is the deviation in the rotor speed, $\Delta \omega$. This signal is often used with a lead compensation type of PSS with a transfer function given by [7]

$$
K(s)=K_{s} \frac{\left(1+s T_{1}\right)^{2}}{\left(1+s T_{2}\right)^{2}} .
$$

The lead compensator structure is quite popular with the industry. Many existing generators are commissioned with a PSS of this form. The tuning procedure for the compensator as suggested in [7] is fairly well understood and is widely used. The output of the PSS, that is, the appropriately phase compensated signal, is fed back at the voltage reference input of the generator AVR to produce the additional rotor damping torque.

For the above controller, the gain $K_{s}$ and the time constants $T_{1}$ and $T_{2}$ are the tunable parameters. The vector of controller parameters is therefore given by $x=\left[\begin{array}{lll}K_{s} & T_{1} & T_{2}\end{array}\right]^{\prime}$. The tuning procedure described in [7] was used to obtain the parameters of the conventional stabilizer for the SMIB model of Fig. 4. This procedure is briefly explained in Appendix II. The system performance with this conventional stabilizer formed the reference set for comparison with the proposed stabilizer.

A robustly $D$-stabilizing controller with minimum gain was designed by solving the following optimization problem.

Find $\left(K_{s}, T_{1}, T_{2}\right)$ s.t. $K_{s}$ is minimized and $g_{i}<0, i=1,2$, $\cdots, n+2$.

Numerous algorithms are available for solving such constrained nonlinear optimization problems [9]. Two different methods have been tried. The first one is based on routines available in the Matlab optimization toolbox and the second one is a simplex method chosen for its ability to handle nonsmooth problems. In both cases, the values of the parameters obtained from the conventional tuning method [7] were taken as the initial guesses. Further, the plant corresponding to the design condition for the conventional PSS was taken as the nominal plant.

\section{A. Nonlinear Programming}

A routine for constrained nonlinear optimization based on sequential quadratic programming, available in the Matlab optimization toolbox was used. The details of the algorithm can be found in [8].

Fig. 6 shows the closed-loop poles for the set $\mathcal{G}$ of plants fitted with this controller. $P, Q$, and $X_{e}$ have been varied with a step size of 0.05 over the specified range. As can be seen from the eigenvalue plots, the closed-loop poles for the entire

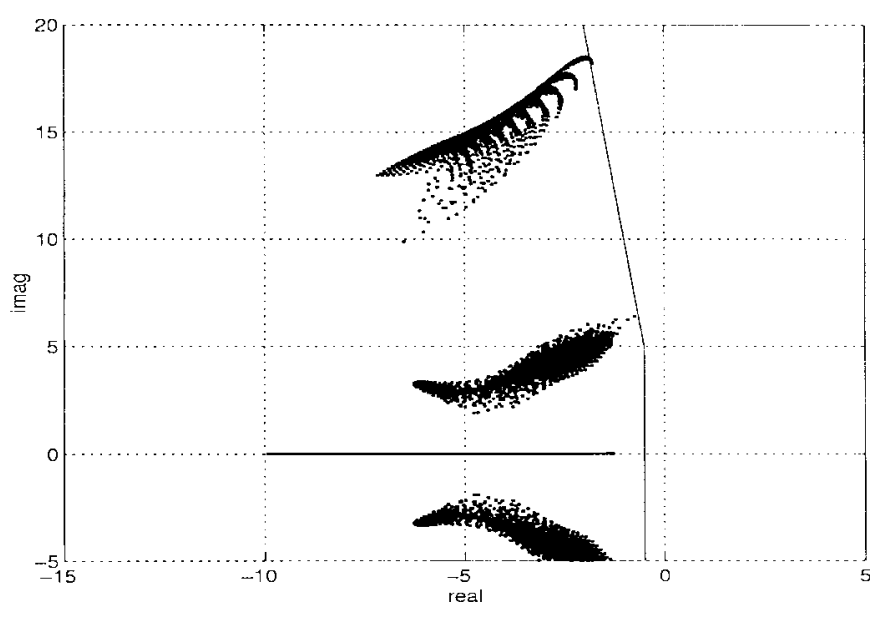

Fig. 6. Closed-loop poles with controller 1.

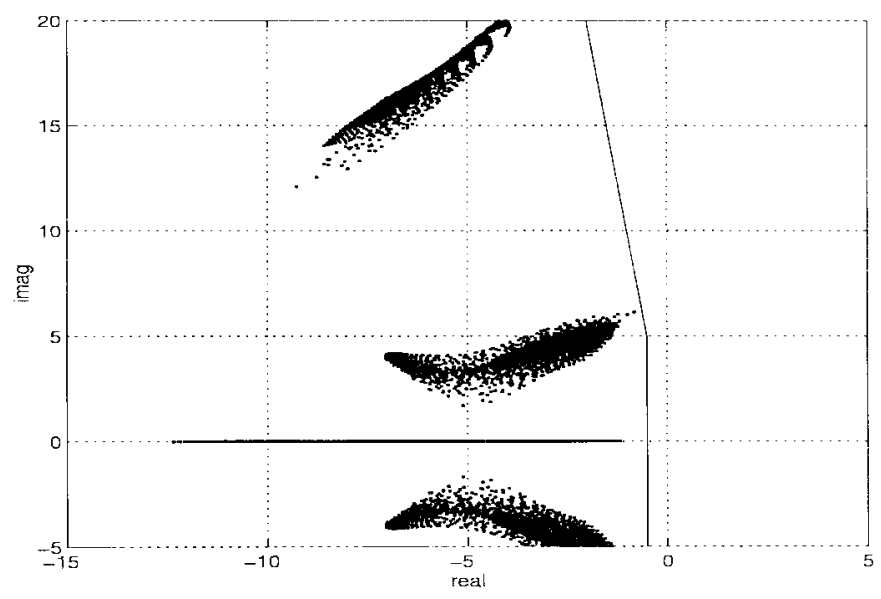

Fig. 7. Closed-loop poles with controller 2.

set lie in an acceptable region, to the left of the $D$-contour, guaranteeing a well damped system response over the chosen range of operating conditions.

\section{B. Simplex Method}

The constraint functions $g_{i}$ were modified to $\max \left(g_{i}, 0\right)$. Then the function " $f$ " defined as

$$
f=K_{s}+\rho \cdot \max _{i}\left(g_{i}\right)
$$

was minimized using Nedler and Mead's simplex algorithm for unconstrained optimization. In the above expression, $\rho$ is a weight that can be adjusted to emphasize the satisfaction of the constraints. As $\rho$ tends to infinity the solution to the above unconstrained problem tends to the solution of the original constrained optimization problem. In this problem a value of $\rho=100$ was found to be satisfactory. Fig. 7 shows the closedloop poles with this controller. Again all the plants in the set $\mathcal{G}$ are seen to be $D$-stable.

The vectors of controller parameters obtained by the conventional and the above two methods are given in Table I. The simplex method is better suited for such nonsmooth problems as it does not involve gradient evaluations but has a slower convergence. 

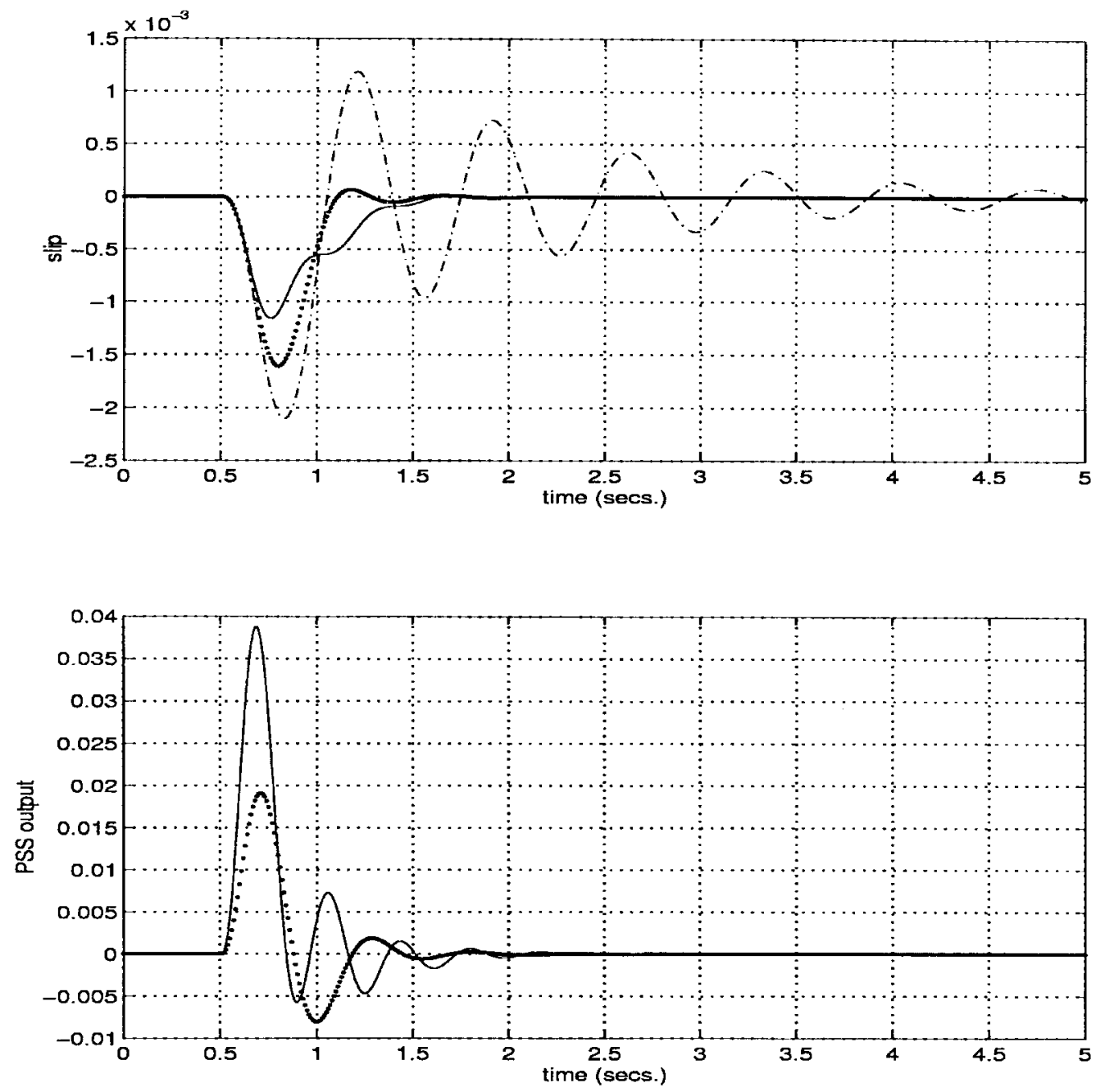

(a)

Fig. 8. System response to a $5 \%$ step disturbance at the voltage reference input of the AVR. (a) $P=0.8, Q=0.4, X_{e}=0.2$. $\cdots$-... No controller, ... conventional, - QFT based.

TABLE I

Computed Controller Parameters

\begin{tabular}{|c|c|c|c|}
\hline Controller & \multicolumn{3}{|c|}{ Design Method } \\
\cline { 2 - 4 } Parameters & Conventional [7] & Non-Lin-Programming & Simplex \\
\hline $\mathrm{K}_{s}$ & 5.5 & 13.88 & 16.22 \\
$\mathrm{~T}_{1}$ & 0.1732 & 0.1785 & 0.1542 \\
$\mathrm{~T}_{2}$ & 0.0577 & 0.0614 & 0.0440 \\
\hline
\end{tabular}

In this particular example, the significant change is in the gain of the controller. The conventional PSS has been tuned to maximize damping. Hence, an increase in the gain results in a reduction in the damping of the nominal plant. In other words, the nominal performance is being detuned for improved robustness. However, it should be noted that any increase in the gain does not necessarily improve robustness. If the gains are further increased beyond the values arrived at by the optimization procedure shown above, some of the operating conditions become unstable. Hence, choice of the gain is quite crucial. This larger gain is acceptable as it does not result in excessive saturation of the stabilizer output as can be seen from the simulation results shown in the next section.
The above optimization problems being nonconvex, global minima cannot be obtained. However, any local minimum close to the initial guess, which we have chosen as the conventional PSS, is acceptable.

The approach to controller synthesis used here is by no means conclusive. Many other optimization methods found suitable for controller design [9] can be used. The formulation in terms of inequalities, i.e., bounds on the frequency response of the loop transfer function is quite general. This formulation is similar to the "method of inequalities" proposed by Zakian [14], [15] for controller design and can be interpreted as an implementation of the moving boundaries algorithm suggested in [14].

The stabilizer was designed to obtain a specified damping performance for the system and the problem of disturbance attenuation was not considered. If required, such specifications could be possibly handled by simultaneously manipulating templates corresponding to points on the $D$-contour, for $D$ stability, and points on the imaginary axis, for shaping the loop transmission function, as in conventional QFT, thereby facilitating the simultaneous handling of both pole region 

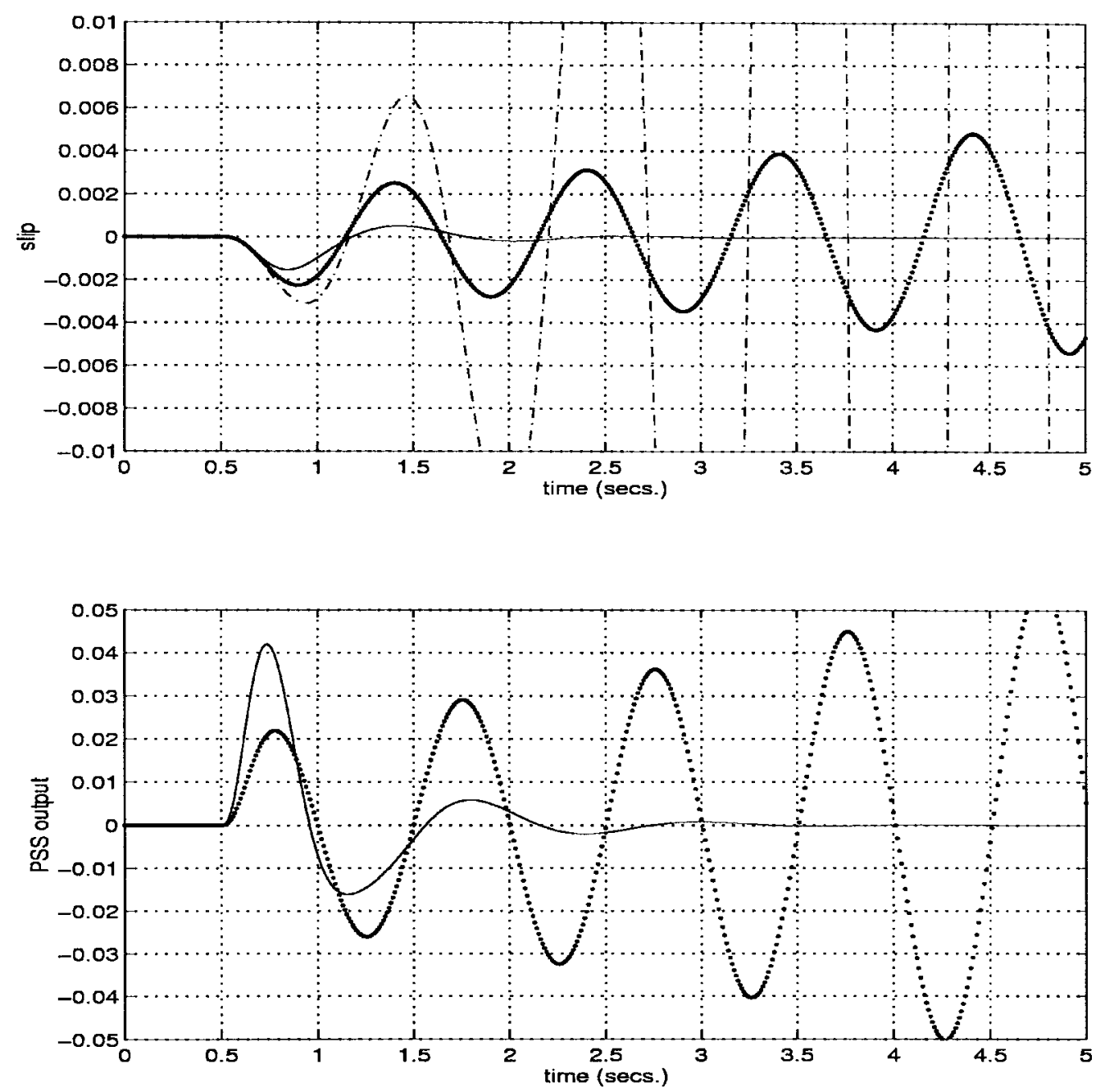

(b)

Fig. 8. (Continued).System response to a $5 \%$ step disturbance at the voltage reference input of the AVR. (b) $P=0.8, Q=0.0, X_{e}=0.6$...$--\cdot$ No controller, ... conventional, - QFT based.

placement as well as closed-loop frequency response specifications.

\section{TIME RESPONSE RESULTS}

The performance of the two controllers was further evaluated by analyzing the system response to a step disturbance injected at the voltage reference input of the AVR, at various operating conditions. Fig. 8(a)-(c) shows the time responses of the uncontrolled plant as well as plants fitted with the conventional and proposed controller 1, at three different operating conditions. Of these, operating condition (a) is the design point for the conventional PSS. As can be expected, the performance of the conventional PSS is quite satisfactory at this operating condition but is unacceptable at the other two system conditions. The conventional stabilizer in fact fails to even stabilize the system at operating condition (b). The QFT based PSS on the other hand performs uniformly well in all the three cases. It is also seen that the frequency of the oscillations has not been altered much by the introduction of the QFT controller and hence there is no loss of synchronizing torque in the system. The outputs of the conventional and the QFT based stabilizers are also shown for each of the three cases. As expected, due to its larger gains, the proposed PSS results in a larger control signal as compared to the conventional one. The control signals do, however, stay within the limits of \pm 0.05 pu which is the hard limit imposed on the output of the PSS in practical implementations. This shows the gain of the proposed PSS to be quite reasonable.

\section{CONCLUSION}

The performance requirements for power system stabilizers have been established in terms of dominant pole region constraints. An effective method, based on QFT, has been presented for tuning the parameters of power system stabilizers. The performance of the resulting robust fixed gain PSS is shown to be satisfactory over a wide range of operating conditions. The conventional lead compensator type of PSS, already available in many power systems, can be simply retuned using the proposed method to achieve enhanced performance. 

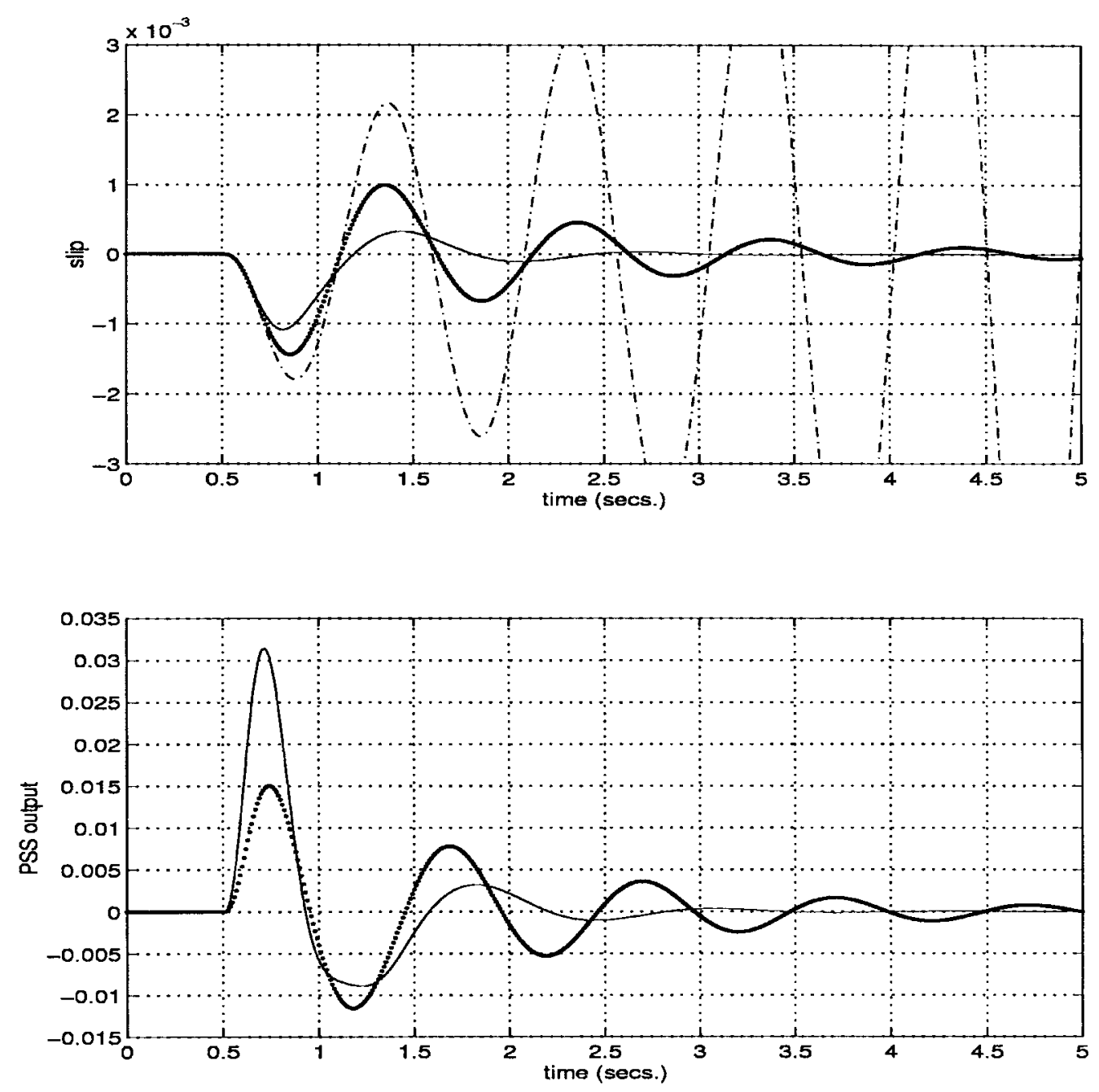

(c)

Fig. 8. (Continued). System response to a $5 \%$ step disturbance at the voltage reference input of the AVR. (c) $P=1.0, Q=0.5, X_{e}=0.7$. -...-. No controller, ... conventional, - QFT based.

APPENDIX I

Single Machine Infinite Bus System

\section{A. Linearized System Equations}

$$
\begin{aligned}
\dot{x}= & {\left[\begin{array}{cccc}
0 & \omega_{B} & 0 & 0 \\
-\frac{K_{2}}{2 H} & -\frac{D}{2 H} & -\frac{K_{1}}{2 H} & 0 \\
-\frac{1}{K_{3} T_{d o}^{\prime}} & 0 & -\frac{K_{4}}{T_{d o}^{\prime}} & \frac{1}{T_{d o}^{\prime}} \\
-\frac{K_{A} K_{6}}{T_{A}} & 0 & -\frac{K_{A} K_{\tilde{b}}}{T_{A}} & -\frac{1}{T_{A}}
\end{array}\right] x } \\
& +\left[\begin{array}{c}
0 \\
0 \\
0 \\
\frac{K_{A}}{T_{A}}
\end{array}\right] V_{\mathrm{REF}}
\end{aligned}
$$$$
\Delta \omega=\left[\begin{array}{llll}
0 & 1 & 0 & 0
\end{array}\right] x
$$

where $x=\left[\begin{array}{llll}\Delta \delta & \Delta \omega & \Delta E_{q}^{\prime} & \Delta E_{F D}\end{array}\right]^{\prime}$.
The constants $K_{1}$ to $K_{6}$ are defined as

$$
\begin{aligned}
& K_{1}=\frac{x_{q}-x_{d}^{\prime}}{x_{e}+x_{d}^{\prime}} i_{q o} E_{b} \operatorname{Sin}\left(\delta_{o}\right)+\frac{E_{q o} E_{b} \operatorname{Cos}\left(\delta_{o}\right)}{x_{e}+x_{q}} \\
& K_{2}=\frac{E_{b} \operatorname{Sin}\left(\delta_{o}\right)}{x_{e}+x_{d}^{\prime}} \\
& K_{3}=\frac{x_{d}^{\prime}+x_{e}}{x_{d}+x_{e}} \\
& K_{4}=\frac{x_{d}-x_{d}^{\prime}}{x_{e}+x_{d}^{\prime}} E_{b} \operatorname{Sin}\left(\delta_{o}\right) \\
& K_{5}=-\left(\frac{v_{d o}}{v_{t o}}\right) \frac{x_{q} E_{b} \operatorname{Cos}\left(\delta_{o}\right)}{x_{e}+x_{q}}-\left(\frac{v_{q o}}{v_{t o}}\right) \frac{x_{d}^{\prime} E_{b} \operatorname{Sin}\left(\delta_{o}\right)}{x_{e}+x_{d}^{\prime}} \\
& K_{6}=\left(\frac{v_{q o}}{v_{t o}}\right) \frac{x_{e}}{x_{e}+x_{d}^{\prime}}
\end{aligned}
$$

where

$\Delta \quad$ small deviation;

$\delta \quad$ rotor angle;

$\omega_{B} \quad$ base speed;

$H \quad$ rotor inertia constant; 
$x_{d}^{\prime} \quad d$-axis transient reactance;

$x_{d}, x_{q} \quad d$ and $q$ axes synchronous reactances;

$i_{d}, i_{q} \quad d$ and $q$ axes generator currents;

$v_{d}, v_{q} \quad d$ and $q$ axes generator voltages;

$v_{t} \quad$ generator terminal voltage;

$E_{q}^{\prime} \quad$ voltage proportional to field flux linkages;

$T_{d o}^{\prime} \quad d$-axis transient open circuit time constant;

subscript $o$ steady state value;

$\omega \quad$ rotor angular speed;

$E_{b} \quad$ infinite bus voltage;

$E_{F D} \quad$ field voltage;

$T_{m} \quad$ mechanical torque;

$T_{e} \quad$ electrical torque;

$K_{A} \quad$ AVR gain;

$T_{A} \quad$ AVR time constant;

$V_{\mathrm{REF}} \quad$ AVR reference input.

\section{B. System Data}

\begin{tabular}{|c|c|c|c|c|c|c|c|c|}
\hline$x_{d}$ & $x_{d}^{\prime}$ & $x_{q}$ & $T_{d o}^{\prime}$ & $E_{b}$ & $H$ & $\omega_{B}$ & $K_{A}$ & $T_{A}$ \\
\hline $2.0 \mathrm{pu}$ & $\begin{array}{c}0.244 \\
\mathrm{pu}\end{array}$ & $\begin{array}{c}1.91 \\
\mathrm{pu}\end{array}$ & $4.18 \mathrm{~s}$ & 1.0 & $3.25 \mathrm{~s}$ & $\begin{array}{c}314.15 \\
\mathrm{rad} / \mathrm{s}\end{array}$ & 50.0 & $0.05 \mathrm{~s}$ \\
\hline
\end{tabular}

\section{APPENDIX II}

The transfer function of the conventional PSS was chosen as

$$
K(s)=K_{s} \frac{(1+0.1732 s)^{2}}{(1+0.0577 s)^{2}} .
$$

The parameters of this lead compensator were tuned for the operating condition $P=0.8, Q=0.4, X_{e}=0.2$ according to the guidelines given in [7].

In this method, first the transfer function of the electrical subsystem is computed. The phase lag of this function at the frequency of oscillation decides the amount of phase lead to be provided by the compensator. $T_{1}$ and $T_{2}$ are chosen to provide the required phase lead at the oscillation frequency. The root locus of the system is then plotted and the gain $K_{s}$ is chosen so as to maximize the damping of the rotor mode.

\section{REFERENCES}

[1] K. E. Bollinger, P. A. Cook, and P. S. Sandhu, "Synchronous generator controller synthesis using moving boundary search techniques with frequency domain constraints," IEEE Trans. Power. Apparat. Syst., vol. 98, pp. 1497-1501, Sept./Oct. 1979.

[2] E. Eitelberg, J. C. Balda, E. S. Boje, and R. G. Harley, "Stabilizing SSR oscillations with a shunt reactor controller for uncertain levels of series compensation," IEEE Trans. Power Syst., vol. 3, pp. 936-943, Aug. 1988.

[3] A. Ghosh, G. Ledwich, O. P. Malik, and G. S. Hope, "Power system stabilizer based on adaptive control technique," IEEE Trans., Power Apparat. Syst. vol. PAS-103, pp. 1983-1989, Aug. 1984.

[4] I. Horowitz, "Survey of quantitative feedback theory," Int. J. Contr., vol. 53, pp. 255-291, 1991.

[5] I. Horowitz and M. Sidi, "Synthesis of feedback systems with large plant ignorance for prescribed time domain tolerances.," Int. J. Contr., vol. 16 , pp. 287-309, 1972.

[6] M. R. Khaldi, A. K. Sarkar, K. Y. Lee, and Y. M. Park, "The modal performance measure for parameter optimization of power system stabilizers," IEEE Trans. Energy Conversion, vol. 8, pp. 660-666, Dec. 1993.

[7] E. V. Larsen and D. A. Swann, "Applying power system stabilizers parts I-III,” IEEE Trans. Power Apparat. Syst., vol. PAS-101, pp. 3017-3046, June 1981.

[8] Matlab Optimization Toolbox-Users' Guide. Natick, MA: The Mathworks, Inc.

[9] D. Q. Mayne and H. Michalska, "Optimization for control design," in CAD for Control Systems, D. A. Linkens, Ed. New York: Marcel Dekker, 1993, pp. 341-366.

[10] K. R. Padiyar, Power System Dynamics, Stability and Control. Singapore: Wiley, 1996

[11] A. J. Urdaneta, N. J. Bacalao, B. Feijoo, L. Flores, and R. Diaz, "Tuning of power system stabilizers using optimization techniques," IEEE Trans. Power Syst, vol. 6, pp. 127-134, Feb. 1991.

[12] L. Wang, "Damping effects of supplementary excitation control signals on stabilizing generator oscillations," Int. J. Electric Power Energy Syst., vol. 18 , no. 1 , pp. $47-53,1996$.

[13] Q. H. Wu, B. W. Hogg, and G. W. Irwin, "A neural network regulator for turboalternators," IEEE Trans. Neural Networks, vol. 3, pp. 95-100, 1992.

[14] V. Zakian and U. Al Naib, "Design of dynamical and control systems by the method of inequalities," Proc. Inst. Elect. Eng., vol. 120, pt. D, no. 11, pp. 1421-1427, Nov. 1973.

[15] V. Zakian, "New formulation for the method of inequalities," Proc. Inst. Elect. Eng., vol. 126, no. 6, pt. D, pp. 579-584, June 1979. 